\title{
PERCEVEJOS PREDADORES (Orius SPP.) (HEMIPTERA: ANTHOCORIDAE) E TRIPES (THYSANOPTERA): INTERAÇÃO NO MESMO HABITAT? ${ }^{1}$
}

Luís Cláudio Paterno Silveira ${ }^{2}$, Vanda Helena Paes Bueno ${ }^{3}$, Júlio Neil Cassa Louzada ${ }^{4}$ e Lívia Mendes Carvalho ${ }^{3}$

\begin{abstract}
RESUMO - O gênero Orius Wolff é composto por espécies de percevejos predadores, principalmente de tripes, encontradas em muitos ecossistemas naturais e manejados. Entretanto, as interações que podem ocorrer entre esses predadores e suas presas no mesmo "habitat" não são bem conhecidas entre as espécies das regiões tropicais. Este estudo teve como objetivo registrar a interação de espécies de Orius e de tripes coletadas na mesma planta, ou seja, presentes no mesmo "habitat". As coletas foram feitas em várias plantas cultivadas, no campo e em casas de vegetação e em plantas invasoras. A forma e o grau de associação entre as espécies foram determinados, utilizando-se o coeficiente de Spearman (R), calculado com dados de presença/ausência das espécies (Orius e tripes) no mesmo "habitat". Orius insidiosus (Say) foi encontrado associado positivamente aos tripes Frankliniella sp., Neohydatothrips sp. e Haplothrips gowdeyi (Franklin) e negativamente associado a Frankliniella schultzei (Trybom) e Caliothrips phaseoli (Hood). Orius thyestes Herring e Orius sp1 ocorreram simultaneamente em 10 espécies de tripes sem, contudo, apresentar associação significativa, enquanto Orius perpunctatus (Reuter) esteve associado positivamente às espécies Frankliniella sp. e Neohydatothrips sp. e, negativamente, a Frankliniella gemina (Moulton).
\end{abstract}

Palavras-chave: Orius insidiosus, pragas, controle biológico e predação.

\section{SPECIES OF ORIUS (HEMIPTERA, ANTHOCORIDAE) AND THRIPS (THYSANOPTERA): INTERACTION IN THE SAME HABITAT?}

\begin{abstract}
The genus Orius Wolff comprise species that are mostly thrip predators, in natural and managed ecosystems. Interactions between Orius species and its preys in the same habitat in tropical areas are not well known. The objective of this research was to record the interactions between Orius and thrips species present in the same habitat. Sampling on crops and weeds were conducted in the field and greenhouse conditions. The association level between Orius and thrips species was evaluated by Spearman coefficient (R) (presence/ absence of the species in the same habitat). Orius insidiosus (Say) was found positively associated with Frankliniella sp., Neohydatothrips sp. and Haplothrips gowdeyi (Franklin), and negatively associated with Frankliniella schultzei (Trybom) and Caliothrips phaseoli (Hood). Orius thyestes Herring and Orius sp1 coexist with 10 thrips species, but no significant association was found between those predators and thrips species. Orius perpunctatus (Reuter) was found positively associated with Frankliniella sp. and Neohydatothrips sp., and negatively associated with Frankliniella gemina (Moulton).
\end{abstract}

Keywords: Orius insidiosus, pests, biological control and predation.

\footnotetext{
${ }^{1}$ Recebido em $1^{\circ} .06 .2004$ e aceito para publicação em 10.08.2005.

${ }^{2}$ Pólo Regional Centro Norte, APTA, Cx. Postal 24, 15830-000 Pindorama-SP. E-mail: <lcpsilveira@ aptaregional.sp.gov.br>.

${ }^{3}$ Universidade Federal de Lavras/Departamento de Entomologia, Cx. Postal 37, 37200-000 Lavras-MG. E-mail:<vhpbueno@ufla.br>.

${ }^{4}$ Universidade Federal de Lavras/Departamento de Biologia, Setor de Ecologia, 37200-000 Lavras-MG. E-mail: <jlouzada@ ufla.br>.
} 


\section{INTRODUÇÃO}

Vários tipos de associações interespecíficas ocorrem entre os seres vivos em um mesmo "habitat", como predação, competição, mutualismo e comensalismo. Segundo Ludwig e Reynolds (1988), a presença ou ausência de espécies no mesmo "habitat" resulta em conclusões importantes a respeito de seu comportamento, como o fato de predadores e presas selecionarem ou evitarem o mesmo "habitat", atraírem-se ou repeliremse mutuamente ou simplesmente não interagirem. Nesse sentido, o estudo de tais associações é de suma importância para os programas de controle biológico aplicado e de conservação de espécies de inimigos naturais.

Espécies do gênero Orius Wolff (Anthocoridae) são onívoras, pois, além de predadores de tripes e outros artrópodes, podem se alimentar de pólen (LATTIN, 2000). São os principais inimigos naturais usados, comercialmente para o controle de Frankliniella occidentalis (Pergande) em casas de vegetação em cultivos de tomate, berinjela, pepino, pimentão e morango, na Europa, EUA e Canadá. Tal sucesso está ligado, entre outros motivos, ao fato de que esse predador e os tripes freqüentemente ocupam os mesmos "microhabitats", como o interior das flores, axilas e meristemas apicais (COOL e RUBERSON, 1998). Essa característica coloca as espécies de Orius como agentes promissores no controle biológico de espécies de tripes-praga (BUENO, 2000).

Riudavets (1995) citou sete espécies do gênero Orius predadoras de F. occidentalis e Thrips tabaci Lind. na Europa, e relatos de Yano (1999) incluíram mais três espécies associadas a Thrips palmi Karny no Japão. No Brasil, a espécie mais abundante é $O$. insidiosus (BUENO, 2000; SILVEIRA et al., 2003). No entanto, segundo Lattin (2000), pouco se conhece sobre as interações desses predadores e suas presas, especialmente das espécies que ocorrem na África e nas Américas Central e do Sul.

O objetivo deste trabalho foi registrar a interação de espécies de Orius e tripes no mesmo "habitat", visando à utilização desses predadores em programas de controle biológico de tripes.

\section{MATERIAL E MÉTODOS}

Os levantamentos das espécies de predadores do gênero Orius e de tripes foram realizados entre os anos de 1999 e 2000 em várias plantas cultivadas e invasoras,

R. Árvore, Viçosa-MG, v.29, n.5, p.767-773, 2005 presentes em condições de campo e em casas de vegetação (Tabela 1), nos municípios de Lavras (MG), Holambra, Campinas e Pindorama (SP).

Nas coletas foi utilizado o método de batida de plantas no interior de sacos plásticos ("tapping method"). Cada amostra foi obtida pela batida de cinco ramos ou inflorescência de determinada planta, sendo posteriormente levada ao laboratório para triagem dos indivíduos. Portanto, cada amostra foi composta, aumentando-se, assim, a representatividade e a chance de se coletarem as principais espécies de Orius e tripes em cada planta amostrada. As espécies de insetos foram separadas inicialmente em morfoespécies e, em seguida, submetidas à maceração, desidratação e montagem em lâmina para identificação. Nos tripes foi utilizada a metodologia proposta por Mound e Kribby (1998) e em Orius, a de Silveira et al. (2003). As identificações dos espécimes foram confirmadas por especialistas dos grupos.

Como não houve padronização do esforço amostral, uma vez que o número e o tamanho das amostras por planta e local não foram constantes, os dados foram analisados pela presença e ausência de Orius e tripes, independentemente da quantidade e do local, da planta e da data de coleta. O total de amostras compostas, somando-se todas as plantas, datas e locais foi $\mathrm{n}=$ 51. Foi denominada a ocorrência simultânea sempre que duas espécies quaisquer de Orius e de tripes foram coletadas juntas numa mesma ocasião. O coeficiente de correlação de Spearman (R) foi utilizado para averiguar se as associações das espécies foram positivas (sinal + ) ou negativas (sinal -), com as probabilidades exatas de erro $(p)$.

\section{RESULTADOS E DISCUSSÃO}

As espécies de predadores encontradas nas amostras foram Orius insidiosus (Say), Orius thyestes (Herring), Orius perpunctatus (Reuter) e Orius sp1. Quanto aos tripes, foram amostrados Frankliniella occidentalis (Pergande), Frankliniella schultzei (Trybom), Frankliniella gemina Bagnall, Frankliniella insularis (Franklin), Frankliniella gardenia (Moulton), Frankliniella condei John, Frankliniella williamsi Hood, Frankliniella sp1, Neohydatothrips sp1, Haplothríps gowdeyi (Franklin), Caliothrips phaseoli (Hood), Thrips palmi Kamy e Thrips australis (Bagnall). Foi coletado também o tripes predador Franklinothrips vespiformis (Crawford) (Tabela 1). 
Percevejos Predadores (Orius Spp.) (Hemptera: ...

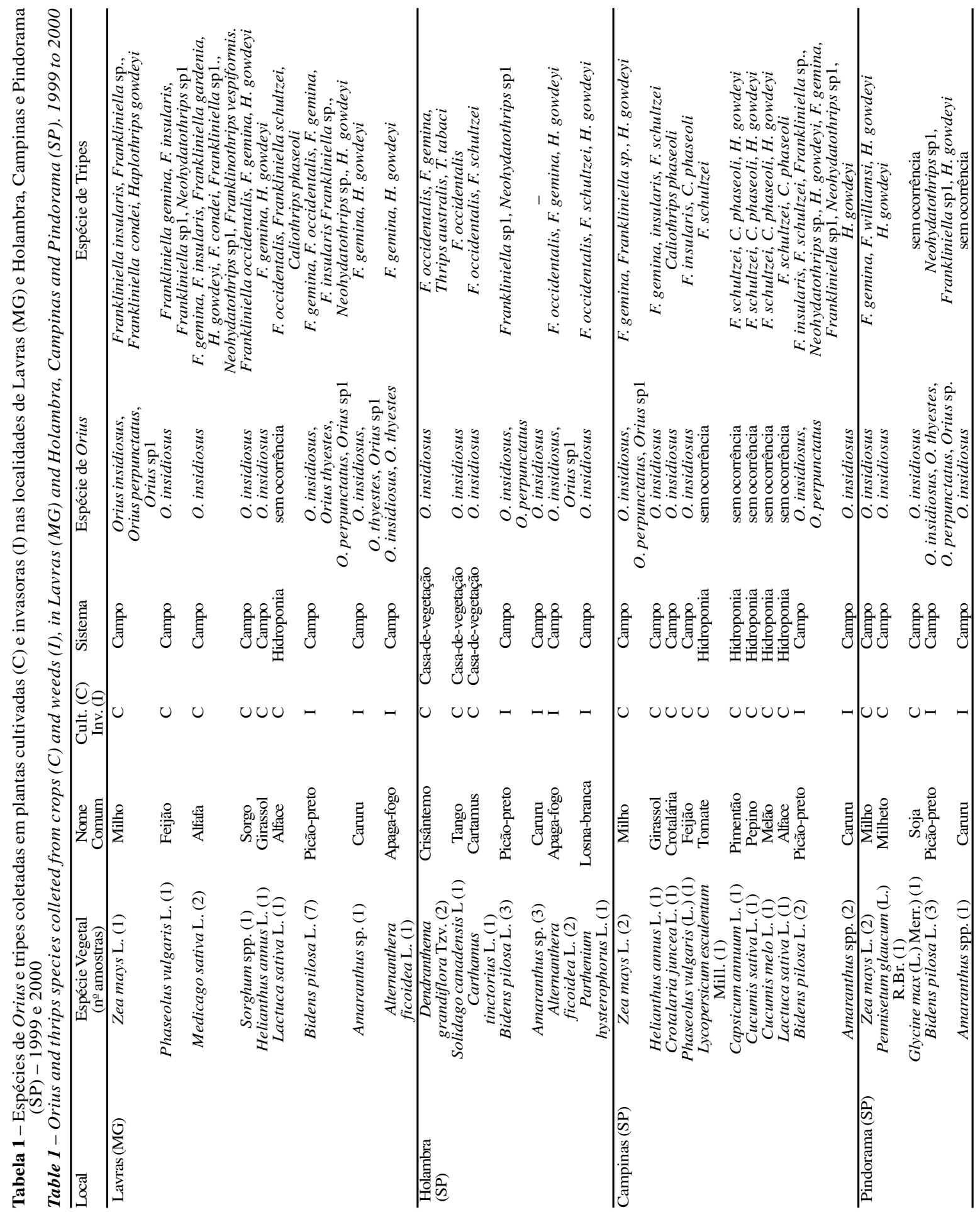

R. Árvore, Viçosa-MG, v.29, n.5, p.767-773, 2005 
O. insidiosus ocorreu simultaneamente em 13 espécies de tripes, em diversas culturas e plantas invasoras, enquanto $O$. thyestes, $O$. perpunctatus e Orius sp1 ocorreram simultaneamente em 10 espécies de tripes, sobretudo no cultivo de milho e nas plantas invasoras (Tabela 1). Essa ocorrência simultânea poderia indicar, em princípio, a existência de associação efetiva (positiva ou negativa) entre predador e presa, porém isso só ocorreu em alguns casos. A análise quanto às associações entre os pares de espécies de Orius e de tripes (Tabela 2) evidenciou que, na maioria das vezes, ocorreu uma associação não-significativa, ou seja, realmente apenas ocorrência simultânea entre espécies.

Associações não-significativas desses predadores também foram verificadas nas espécies de tripes $F$. occidentalis, T. palmi e T. australis, consideradas pragas de importância agrícola, demonstrando que o fato de terem sido coletados juntos em alguma ocasião (Tabela 1) não indicou que o predador estivesse realmente associado a essas presas, mas sim que podem ter sido coletados juntos devido ao acaso. Essas espécies de tripes foram comuns em coletas em cultivos de flores em casas de vegetação (Holambra, SP), sobretudo em plantas de áreas de descarte ou de áreas abandonadas, sem ter havido a colheita, onde também foram encontrados predadores do gênero Orius. Entretanto, nas coletas nessa localidade (Holambra,SP), feitas em cultivos com intensas aplicações de produtos fitossanitários, não foram encontradas espécies de Orius, mas sim de tripes, podendo-se supor que a aplicação desses produtos nesses cultivos tenha influenciado a não-ocorrência desses predadores e a possibilidade de uma associação efetiva entre aquelas espécies de tripes e de Orius. Segundo van Lenteren (2000), muitos parasitóides e predadores que ocorrem naturalmente no campo podem migrar para o interior de casas de vegetação e se estabelecerem nesses ambientes, desde que condições adequadas estejam presentes.

Com o tripes predador $F$. vespiformis, que explora os mesmos tipos de presas que as espécies do gênero Orius (JOHANSEN, 1976; LOOMANS e VIERBERGEN, 1999), também não foi encontrada associação significativa dessas espécies de Orius amostradas (Tabela 3), o que nesse caso diminui a possibilidade da ocorrência de predação intraguilda e competição entre esses predadores nos mesmos "habitats" onde estavam presentes. Segundo Schmidt et al. (1998), Albajes e Alomar (1999) e Brodeur et al. (2002), a predação intraguilda, isto é, quando ocorre predação mútua de organismos que exploram a mesma presa, pode interferir desfavoravelmente em programas de controle biológico.

Tabela 2 - Nível de associação entre espécies de Orius e tripes coletadas no mesmo "habitat", pelo Coeficiente de Correlação de Spearman $(\mathrm{R})$, e com as probabilidades exatas de erro $(\mathrm{n}=51)-1999$ a 2001

Table 2 -Association level between the species Orius and thrips collected in the same habitat, by Spearman Correlation Coefficient $(R)$ and with the exact error probabilities $(n=51)-1999$ to 2000

\begin{tabular}{|c|c|c|c|c|c|c|c|c|}
\hline \multirow{2}{*}{$\begin{array}{l}\text { Espécies } \\
\text { Tripes }\end{array}$} & \multicolumn{2}{|c|}{ Orius insidiosus } & \multicolumn{2}{|c|}{ Orius perpunctatus } & \multicolumn{2}{|c|}{ Orius thyestes } & \multicolumn{2}{|c|}{ Orius sp1 } \\
\hline & $\mathrm{R}$ & $p^{(1)}$ & $\mathrm{R}$ & $p$ & $\mathrm{R}$ & $P$ & $\mathrm{R}$ & $p$ \\
\hline Frankliniella $\mathrm{sp} 1$ & 0,3731 & 0,0097 & 0,5078 & 0,0002 & 0,1376 & n.s. & 0,2045 & n.s. \\
\hline Neohydatothrips sp1 & 0,3253 & 0,0256 & 0,4561 & 0,0012 & 0,0433 & n.s. & 0,1288 & n.s. \\
\hline H. gowdeyi & 0,3641 & 0,0086 & 0,0561 & n.s. & 0,2434 & 0,0851 & 0,2696 & 0,0556 \\
\hline F. gemina & 0,2753 & 0,0611 & $-0,3601$ & 0,0129 & 0,1217 & n.s. & $-0,2002$ & n.s. \\
\hline C. phaseoli & $-0,7776$ & $1,93 \times 10^{-11}$ & $-0,1720$ & n.s. & $-0,1422$ & n.s. & $-0,1575$ & n.s. \\
\hline F. schultzei & $-0,4180$ & 0,0022 & 0,1589 & n.s. & $-0,0415$ & n.s. & 0,2053 & n.s. \\
\hline F. occidentalis & 0,0062 & n.s. & $-0,2333$ & n.s. & $-0,0415$ & n.s. & $-0,0739$ & n.s. \\
\hline F. condei & 0,0955 & n.s. & $-0,0882$ & n.s. & $-0,0727$ & n.s. & $-0,0806$ & n.s. \\
\hline F. gardenia & 0,0954 & n.s. & $-0,0881$ & n.s. & $-0,0727$ & n.s. & $-0,0806$ & n.s. \\
\hline F. williamsi & 0,0667 & n.s. & $-0,0616$ & n.s. & $-0,0508$ & n.s. & $-0,0564$ & n.s. \\
\hline F. insularis & 0,0545 & n.s. & $-0,0304$ & n.s. & 0,0273 & n.s. & $-0,1732$ & n.s. \\
\hline T. australis & 0,0667 & n.s. & $-0,0616$ & n.s. & $-0,0508$ & n.s. & $-0,0564$ & n.s. \\
\hline T. palmi & 0,0667 & n.s. & $-0,0616$ & n.s. & $-0,0508$ & n.s. & $-0,0564$ & n.s. \\
\hline F. vespiformis & 0,0667 & n.s. & $-0,0616$ & n.s. & $-0,0508$ & n.s. & $-0,0564$ & n.s. \\
\hline
\end{tabular}

${ }^{1}$ Valores de probabilidade de erro $p$ acima de 0,10 foram considerados não-significativos (n.s.).

R. Árvore, Viçosa-MG, v.29, n.5, p.767-773, 2005 
Os tripes Frankliniella sp1, Neohydatothrips sp1 e H. gowdeyi foram coletados em várias plantas, mas principalmente naquelas invasoras e no cultivo de milho (Tabela 1). Os valores de $\mathrm{R}$ nessas espécies de tripes em relação a $O$. insidiosus e $O$. perpunctatus foram mais altos que os demais (entre 0,32 e 0,50), além de significativos (Tabela 2). Esse fato pode indicar que essas espécies de tripes estão associadas positivamente àquelas de Orius nos "habitats" amostrados. Tal associação positiva abre precedentes para estudos mais conclusivos quanto à presença dos predadores nessas plantas e cria perspectivas para a utilização destas como reservatórios naturais de predadores do gênero Orius em sistemas de conservação em programas de controle biológico. Como essas plantas hospedam, sobretudo, espécies de tripes que não apresentam o "status" de pragas, elas poderiam ser mantidas no ambiente, de modo a hospedar diferentes espécies de Orius próximas às culturas de interesse econômico. Elkassabany et al. (1996) verificaram que $O$. insidiosus foi coletado em várias plantas invasoras, quando estas estavam em floração e hospedando altas densidades de tripes. Também, a utilização de plantas hospedeiras para predadores teve sua eficiência comprovada por Arnó et al. (2000), os quais distribuíram plantas de fumo dentro de casas de vegetação com plantio de tomate para que o percevejo predador Macrolophus caliginosus Wagner (Heteroptera: Miridae) pudesse se manter à custa de presas alternativas e migrar para as plantas de tomate, de modo a controlar a moscabranca (Bemisia spp.). O resultado satisfatório obtido por esses autores indica que essa técnica é viável e pode ser adaptada a outras situações de interesse.

Plantas invasoras onde foram coletadas espécies de tripes neste estudo (Tabela 1) poderiam, assim, ser mantidas em faixas intercalares, nas bordaduras dos plantios, em ilhas de vegetação ou nas áreas de pousio, com o intuito de promover o estabelecimento de espécies de tripes não-pragas e, conseqüentemente, de espécies de Orius associadas a elas. No interior de casas de vegetação, essas plantas também poderiam ser utilizadas em posições estratégicas como plantas banqueiras ou unidades de criação aberta para tripes não-pragas do cultivo principal de Orius.

Além das associações não-significativas e das positivas, foram observadas associações negativas entre as espécies de Orius e de tripes (Tabela 2), as quais foram significativas para C. phaseoli e F. schultzei em relação a $O$. insidiosus e para $F$. gemina em relação a $O$. perpunctatus. Nas duas primeiras espécies de tripes, comuns nas coletas em plantas sob o sistema de hidroponia (Tabela 1), este resultado era esperado, pois não foram coletados predadores do gênero Orius nesse sistema de cultivo em nenhuma ocasião. Tal fato pode estar relacionado, ao menos parcialmente, ao uso de produtos químicos de amplo espectro de ação e não seletivos a inimigos naturais nos cultivos amostrados. Em razão disso, a ocorrência de tripes em plantas em culturas hidropônicas pode significar uma adaptação a um ambiente não explorado por seus predadores. Essa constatação sustentaria a hipótese da exploração de espaços livres de inimigos, formulada por Lawton (1983) e Jeffries e Lawton (1984), segundo a qual os organismos fitófagos procuram nas plantas dentro de determinado "habitat" maneiras e, ou, locais para escaparem de seus inimigos naturais, ou seja, locais que são favoráveis aos herbívoros, mas não aos agentes entomófagos.

As associações positivas ou negativas, verificadas entre espécies de Orius e tripes nesse levantamento, reveladas pelo coeficiente de Spearman (R), apesar de significativas, não permitem concluir sobre que tipo de relação ecológica essas espécies mantêm entre si, ou seja, se ocorre apenas a predação de Orius sobre tripes ou se também há competição por alimento (pólen) e,ou, abrigo. Segundo Venzon (2000), várias dessas associações podem ocorrer conjuntamente, sendo, entretanto, difícil separá-las quando várias espécies de herbívoros e de predadores onívoros são encontradas habitando uma mesma planta, pois as teias alimentares se tomam complexas.

No entanto, apesar dessa complexidade nas associações, tais informações abrem boas perspectivas para estudos futuros, sobretudo em relação ao manejo de plantas invasoras ou cultivadas para manutenção desses predadores nos agroecossistemas, além daqueles relacionados à ausência de insetos predadores em determinados sistemas de cultivo, sobretudo pelos efeitos nocivos da aplicação indiscriminada de produtos fitossanitários. As diferentes espécies de Orius e tripes ocorrem simultaneamente em diversas plantas no mesmo "habitat", e esse conhecimento deve ser utilizado de modo a aumentar a sustentabilidade dos programas de controle biológico, bem como para reduzir o seu custo.

R. Árvore, Viçosa-MG, v.29, n.5, p.767-773, 2005 


\section{AGRADECIMENTOS}

À Renata C. Monteiro, pela identificação das espécies de tripes; a Maria G. Tommasinni (CRPV, Itália), Luciana Tavella (Universitá di Torino, Itália) e Diego Carpintero (Museo de La Plata, Argentina), pela identificação das espécies do gênero Orius; e ao CNPq, pelas bolsas de estudo concedidas aos primeiros autores, bem como pelo suporte financeiro à realização deste trabalho.

\section{REFERÊNCIAS BIBLIOGRÁFICAS}

ALBAJES, R.; ALOMAR, O. Current and potential use of poliphagous predators. In: ALBAJES, R.(Eds.). Integrated pest and disease management in greenhouse crops. Dordrecht: Kluwer Academic Publishers, 1999, p. 265-275.

ARNÓ, J. et al. Conservation of Macrolophus caliginosus Wagner (Het. Miridae) in

commercial greenhouses during tomato crop-free periods. IOBC/WPRS Bulletin, v. 23, n. 1, p. 241-246, 2000.

BUENO, V.H.P. (Ed.). Controle biológico de pragas: produção massal e controle de qualidade. Lavras: Universidade Federal de Lavras, 2000. 207p.

BRODEUR, J.; CLOUTIER, C.; GILLESPIE, D. High-order predators in greenhouse systems. IOBC/WPRS Bulletin, v. 25, p.33-36, 2002.

COOL, M.; RUBERSON, J. R. Predatory Heteroptera: their ecology and use in biological control. Proceedings Annual Meetings Entomolology Society of America, Indianapolis: 1993. 1998. 233 p.

ELKASSABANY, E.; RUBERSON, J. R.; KRING, T. Seasonal distribution and overwintering of Orius insidiosus (Say) in Arkansas. Journal of Entomological Science, v. 31, n. 1, p. 76-88, 1996.

JEFFRIES, M.J.; LAWTON, J.H. Enemy free space and the structure of ecological communities. Biological Journal of Linnean Society, v. 23, p. 269-286, 1984.

R. Árvore, Viçosa-MG, v.29, n.5, p.767-773, 2005
JOHANSEN, R.M. Algunos aspectos sobre la conducta mimética de Franklinothrips vespiformis (Crawford) (Insecta: Thysanoptera). An. Inst. Universidad Nacional Autónoma de México, v. 47, p. 25-50, 1976.

LATTIN, J.D. Economic importance of minute pirate bugs (Anthocoridae). In.: SCHOEFER, C.W.; PANIZZI, A.R. (Eds.). Heteroptera of economic importance. Flórida: CRC Press, 2000. 828p.

LAWTON, J.H. Plant architecture and the diversity of phytofagous insects. Annual Review of Entomology, v. 28, p. 23-39, 1983.

LOOMANS, A.J.M.; VIERBERGEN, G.

Franklinothrips: perspectives for greenhouse pest control. IOBC/WPRS Bulletin, v. 22, p. $157-160,1999$.

LUDWIG, J.A.; REYNOLDS, J.F. Statistical ecology: a primer on methods and computing. New York: John Wiley, 1988. 337 p.

MOUND, L.A.; KRIBBY, G. Thysanoptera An identification guide. 2. ed.Wallingford: CAB International, 1998. 70p.

RIUDAVETS, J. Predator of Frankliniella occidentalis (Perg.) and Thrips tabaci Lind.: a review. Waegeningen Agricultural University Papers, v. 95, p. 43-87, 1995.

SCHMIDT, J. M.; TAYLOR, J. R.; ROSENHEIM, J. A. Canibalism and intraguild predation in the predatory heteroptera. In: ANNUAL MEETING OF THE ENTOMOLOGICAL SOCIETY OF AMERICA, 1998, Lanham. Proceedings.... Lanhan: 1998. p. 131-169.

SILVEIRA, L.C.P.; BUENO, V.H.P.; MENDES, S.M. Record of two species of Orius Wolff (Hemiptera, Anthocoridae) in Brazil. Revista Brasileira de Entomologia, v. 47, p. 303-306, 2003.

Van LENTEREN, J. C. Critérios de seleção de inimigos naturais a serem usados em programas de controle biológico. In.: BUENO, V.H.P. (Ed.). Controle biológico de pragas: produção massal e controle de qualidade. Lavras: Universidade Federal de Lavras, 2000. 207 p. 
VENZON, M. Food webs on plants: the role of a generalist predator. 2000. $91 \mathrm{f}$. Thesis (Ph.D. in Entomology) - University of Amsterdam, Amsterdam, 2000.
YANO, E. Recent advances in the study of biocontrol with indigenous natural enemies in Japan. IOBC/WPRS Bulletin, v. 22, p.291-294, 1999. 